Document downloaded from:

http://hdl.handle.net/10251/148457

This paper must be cited as:

Oroval, M.; Díez, P.; Aznar, E.; Coll Merino, MC.; Marcos Martínez, MD.; Sancenón Galarza, F.; Villalonga, R.... (2017). Self-Regulated Glucose-Sensitive Neoglycoenzyme-Capped Mesoporous Silica Nanoparticles for Insulin Delivery. Chemistry - A European Journal. 23(6):1353-1360. https://doi.org/10.1002/chem.201604104

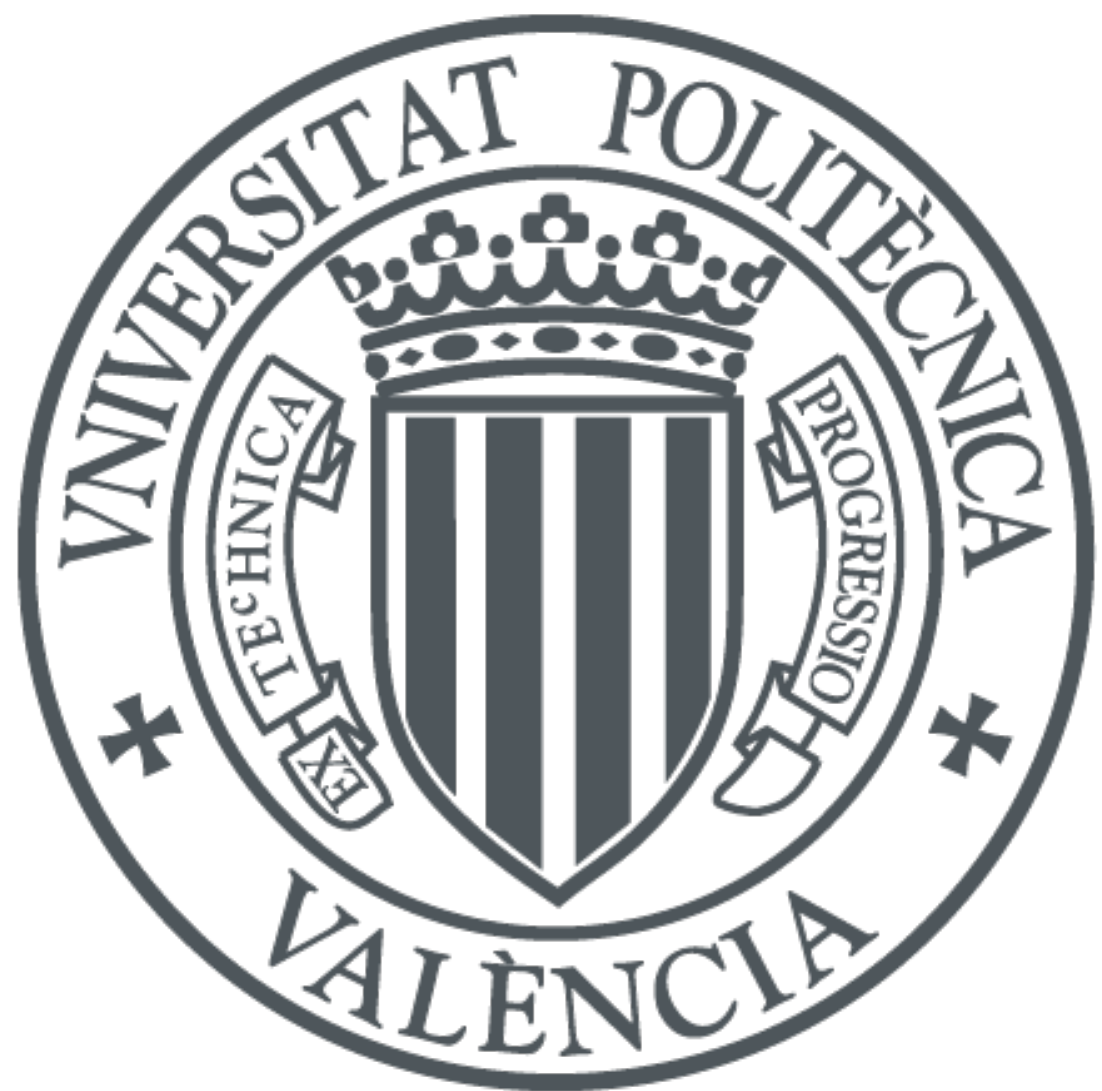

The final publication is available at

https://doi.org/10.1002/chem.201604104

Copyright John Wiley \& Sons

Additional Information

"This is the peer reviewed version of the following article: Oroval, Mar, Paula Díez, Elena Aznar, Carmen Coll, María Dolores Marcos, Félix Sancenón, Reynaldo Villalonga, and Ramón Martínez-Máñez. 2016. Self-Regulated Glucose-Sensitive NeoglycoenzymeCapped Mesoporous Silica Nanoparticles for Insulin Delivery. Chemistry - A European Journal 23 (6). Wiley: 1353 60. doi:10.1002/chem.201604104, which has been published in final form at https://doi.org/10.1002/chem.201604104. This article may be used for noncommercial purposes in accordance with Wiley Terms and Conditions for Self-Archiving." 


\title{
Self-regulated glucose-sensitive neoglycoenzyme-capped mesoporous silica nanoparticles for insulin delivery
}

\author{
Mar Oroval, ${ }^{[a],[b]}$ Paula Díez , ${ }^{[c],[d]}$ Elena Aznar, ${ }^{[b],[a]}$ Carmen Coll, ${ }^{[a],[b]}$ María Dolores Marcos, ${ }^{[a],[b],[e]}$ Félix \\ Sancenón, ${ }^{[a],[b],[e]}$ Reynaldo Villalonga ${ }^{[c],[d]}$ and Ramón Martínez-Máñez[a],[b],[e]
}

\begin{abstract}
We describe herein the preparation of glucose-sensitive capped mesoporous silica nanoparticles for insulin delivery. The new material consists of an expanded pore nanometric silica support grafted with 1-H-propyl-1-H-benzimidazole groups, loaded with fluorescein isothiocyanate-labeled insulin (FITC-Ins) and capped by the formation of inclusion complexes between CD-modified-glucose oxidase (CD-GOx) and the benzimidazole groups grafted on the mesoporous support. Insulin delivery from the gated material in simulated blood plasma was assessed upon addition of glucose. Glucose is transformed by GOx into gluconic acid that promoted the dethreating of the benzimidazole-CD-GOx inclusion complexes allowing cargo release. Small quantities of this support would be needed to release the amount of insulin necessary to decrease diabetic blood glucose concentration to the regular level.
\end{abstract}

\section{Introduction}

Hybrid organic-inorganic nanomaterials have attracted significant interest due to their unique properties resulting from the combination of organic and bioorganic chemistry and material science. ${ }^{[1]}$ In this field, the design and synthesis of mesoporous hybrid gated materials, with the ability to be selectively opened upon the application of an external stimulus and release an entrapped cargo, has generated special interest to the scientific community in recent years. ${ }^{[2-5]}$ These gated materials are composed of two main subunits: (i) a porous scaffold in which a certain cargo is stored and (ii) molecular, supramolecular or biomolecular entities grafted onto the outer surface, which allow control delivery of the entrapped payload. ${ }^{[6,7]}$ Mesoporous silica nanoparticles (MSNs) have been widely used as inorganic support in the construction of gated nanodevices. MSNs present some remarkable features as they are mechanically hard, biocompatible, ease to functionalize using well-known chemistries, have a high loading capacity and

[a] M. Oroval, Dr. E. Aznar, Dr. C. Coll, Dr. M.D Marcos, Dr. F. Sancenón, Prof. R. Martínez-Máñez

Instituto Interuniversitario de Investigación de Reconocimiento Molecular y Desarrollo Tecnológico. Unidad Mixta Universitat Politècnica de València - Universitat de València. Camino de Vera s/n, 46022, Valencia, Spain

[b] M. Oroval, Dr. E. Aznar, Dr. M.D Marcos, Dr. F. Sancenón, Prof. R. Martínez-Máñez

CIBER de Bioingeniería, Biomateriales y Nanomedicina (CIBER-BBN)

[c] P Díez, Dr. R. Villalonga

Department of Analytical Chemistry, Faculty of Chemistry, Universidad Complutense de Madrid, Madrid (Spain)

[d] P Díez, Dr. R. Villalonga

IMDEA Nanoscience, Cantoblanco Universitary city, Madrid (Spain)

[e] Dr. M.D Marcos, Dr. F. Sancenón, Prof. R. Martínez-Máñez Departamento de Química, Universitat Politècnica de València, Camino de Vera s/n, 46022 València, Spain tunable pore size. ${ }^{[8-10]}$ In fact, numerous controlled-release systems using mesoporous silica supports have been described using physical, ${ }^{[11-14]}$ chemical| ${ }^{[15-17]}$ or biochemical ${ }^{[18-21]}$ stimuli.

From different point of view, diabetes mellitus is a chronic metabolic disease characterized by an accumulating concentration of glucose in blood and urine. This illness, which requires ongoing medical care and patient self-management, takes place when beta cells of pancreas fail to produce insulin (type 1) or produce it insufficiently to overcome insulin resistance in target tissues (type 2). ${ }^{[22]}$ Moreover, it is a major leading cause of mortality in industrialized countries, leading to severe complications, such as kidney disease, retinopathy, neuropathy, leg or foot amputations and heart diseases. ${ }^{[23]}$ In healthy humans, the concentration of glucose in blood is controlled in a finely regulated manner to maintain glucose between 3.5 and $7 \mathrm{mM}^{[24]}$ In diabetic patients, administration of insulin to help the body to use or store the blood glucose obtained from food is essential in type 1 and longstanding type 2 diabetes patients, requiring continuous dose adjustments based on self-monitoring of blood glucose levels. This widely used therapy is not exempt of hazards. The most important risk when treating with insulin is hypoglycemia. ${ }^{[25]}$ Extremely low blood sugar levels can be life-threatening resulting in seizures, nervous system damage and even comma. Thus, a perfect insulin therapy should minimize the risk of hypoglycemia while improving glycemic control. In this context, the design systems able to detect fluctuations in blood glucose concentrations and release insulin in a self-regulated fashion is highly desirable and still a challenge. ${ }^{[26-28]}$

In this context different strategies integrated with a large variety of chemical approaches for insulin delivery in response to glucose concentration changes have been reported. ${ }^{[29-39]}$ Nevertheless, only few MSNs based systems have been described for this purpose. One of the first reported examples was developed by Lin et al., ${ }^{[40]}$ that used MSNs capped with gluconic acid-modified insulin (G-Ins) and loaded with cyclic adenosine monophosphate (CAMP). The nanomaterial demonstrated to be glucose-sensitive, releasing both G-Ins and cAMP. Moreover, Shi and co-workers reported an insulin loaded mesoporous silica particles coated with GOx enzyme-multilayers that were cross-linked with glutaraldehyde and acted as gatekeepers. In the presence of glucose, GOx generated gluconic acid with a subsequent decrease in the environment $\mathrm{pH}$. As a consequence, the enzyme multilayer was expanded and the entrapped insulin released. ${ }^{[41]}$ Another example was recently developed by Asefa and co-workers who described the preparation of MSNs for insulin release by tethering insulin molecules onto boronic acid functionalized MSNs and then coating the material with a shell of the $\mathrm{pH}$-sensitive polymer polyacrylic acid. ${ }^{[42]}$ The material exhibited both $\mathrm{pH}$ - and glucosedependent release of insulin. 
Based in these concepts and considering the multiple advantages of MSNs as carriers in controlled release applications, ${ }^{[43-45]}$ we report herein the design of a new selfregulated glucose-sensitive platform for insulin release. Our strategy relies on the use of $\beta$-cyclodextrin-modified enzyme glucose oxidase (CD-GOx) as gatekeeper in MSNs and the enzymatic reaction between glucose and GOx as trigger which induces insulin delivery. ${ }^{[46]}$ This approach is simple and present some advantages versus other reported glucose-triggered insulin release, such as the storage of insulin into the pores preventing its degradation.

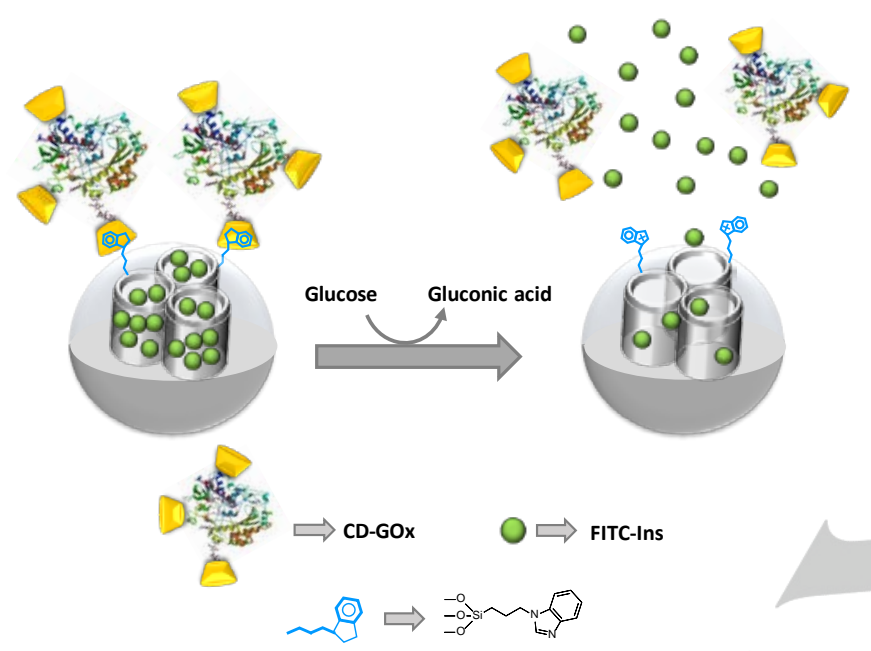

Scheme 1. Schematic representation of the glucose-triggered FITC-Ins release from nanodevice $\mathbf{S 3}$.

\section{Results and Discussion}

\section{The glucose-responsive nanomaterial.}

The designed nanodevice for controlled insulin release is shown in Scheme 1. The system consists of MSNs with large pores loaded with fluorescein isothiocyanate-labeled insulin (FITC-Ins), functionalized on the outer surface with 1-propyl-1-Hbenzimidazol groups and capped by CD-GOx via the formation of inclusion complexes between benzimidazol groups in the pore outlets and $\beta$-cyclodextrin groups from CD-GOx (solid S3). When $\mathbf{S} \mathbf{3}$ is placed in an aqueous medium, addition of glucose is expected to trigger insulin delivery. Glucose is recognized by glucose oxidase enzyme and hydrolyzed into gluconic acid $\left(\mathrm{pK}_{\mathrm{a}}=3.6\right)$. The generation of gluconic acid induces a local drop in $\mathrm{pH}$ that would cause the protonation of benzimidazol groups $\left(\mathrm{pK}_{\mathrm{a}}=5.55\right)$ on $\mathrm{S} 3$ and the dethreading of the inclusion complexes between benzimidazol and CD-GOx, finally resulting in the delivery of entrapped insulin yielding solid S4.

MSNs with large pores were prepared by the two-step swelling incorporation method, following previous reports with some modifications. ${ }^{[47,48]}$ Especifically, tetramethylorthosilicate (TMOS) and cetyltrimethylammonium bromide (CTAB) were used as the inorganic precursor and structure directing agent, respectively to obtain a nanoparticulated and mesostructured material (S0-1). In a second step, material S0-1 was subjected to a hydrothermal treatment in the presence of equal volumes of 1,3,5-trimethylbenzene (TMB), water, and ethanol to achieve the pore expansion (S0-2). Then the surfactant and the pore expanding agent (TMB) were removed by calcination to obtain the final support S0-3. The calcined nanoparticles were grafted with 3-iodopropyltrimethoxysilane and the resulting material was treated with benzimidazole yielding solid S1 which contains 1propyl-1-H-benzimidazole groups in the surface. Subsequently, the pores were loaded with FITC-Ins by a diffusion process to give solid S2. Finally, the support was capped by the addition of CD-GOx to a suspension of $\mathbf{S} 2$ in water at $\mathrm{pH} 7.5$ to yield the final capped solid S3, which was centrifuged and carefully washed with water at $\mathrm{pH} 7.5$.

\section{Materials characterization.}

The prepared materials were characterized using standard procedures. The powder X-ray diffraction (PXRD) pattern of solids S0-1, S0-2, S0-3, S1 and S2 are shown in Figure 1. As observed, the PXRD pattern of the as-synthesized nanoparticles s0-1 (Figure 1, curve a) shows the three typical low-angle reflections of a hexagonal-ordered matrix that can be indexed as (100), (110) and (200) Bragg peaks. However, the regularity of mesopores was highly diminished after pore expansion due to the reorganization of the internal mesostructure, which is corroborated by the significant broadening and the clearly observed displacement of the (100) peak to lower $2 \theta$ values $\left(0.976^{\circ}\right)$ (Figure 1, curve b, solid S0-2). In addition, diffraction peaks assignable to the (110) and (200) planes disappeared. On the other hand, the condensation of silanol groups during the calcination step is reflected in a slight shift of the (100) peak to higher $2 \theta$ values $\left(1.094^{\circ}\right)$ (Figure 1 , curve $c$, solid $\mathbf{s} 0-3$ ), corresponding to an approximate cell contraction of $11.3 \AA$. The $\mathrm{a}_{0}$ cell parameter for $\mathbf{S 0 - 3}$ was calculated to be $93.13 \AA\left(d_{100}=\right.$ $80.65 \AA$ ). Finally, curves $d$ and e in Figure 1 , correspond to the PXRD pattern of S1 and S2. The presence of the (100) peak in these solids indicated that besides surface functionalization and pore loading, the porous structure in the nanoparticles is preserved.

By means of transmission electron microscopy (TEM) analysis the porous structure, particle size and morphology of the different samples was also studied. Figure 2 shows a representative image of the calcined scaffold S0-3, S1, S2, S3 and $\mathbf{S} 4$ materials. It can be clearly seen that these nanoparticles show a spherical form with diameters ranging from 200 to 300 $\mathrm{nm}$. TEM images of S1, S2 and S3, also evidence that porosity is still retained after functionalization with 1-propyl-1-Hbenzimidazole, loading with FITC-Ins and capping with CD-GOx (Figure $2 \mathrm{~b}, \mathrm{c}, \mathrm{d}$ ). Moreover, the TEM representative image of solid S4 (Figure 2f) confirms that the particle morphology and the porous network remained unaltered after delivery of FITCIns and the detachment of CD-GOx from the surface. In addition, particle size and morphology of solid S3 was also studied by field emission scanning electron microscopy (FSEM). Figure 2e confirms the spherical morphology and size of solid $\mathbf{S} 3$ observed in TEM analysis (Figure $2 \mathrm{~d}$ ). 


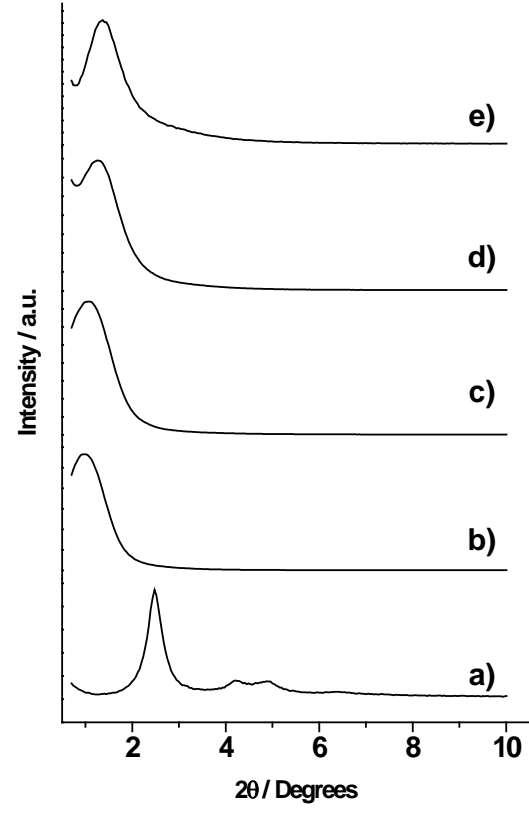

Figure 1. Powder X-ray diffraction patterns of a) as synthesised nanoparticles S0-1, b) pore expanded nanoparticles S0-2, c) pore expanded and calcined nanoparticles S0-3, d) solid S1 containing 1-propyl-1-H-benzimidazole and e) solid S2 containing 1-propyl-1-H-benzimidazole and loaded with FITC-Ins.

As a complement to PXRD and TEM, adsorptiondesorption measurements were also carried out to study the porous structure of the calcined nanoparticles S0-3 and solid S2 (see Figure 3). Using the Barret-Joyner-Halenda $(\mathrm{BJH})^{[49]}$ model on the adsorption branch of the isotherm the pore diameter and pore volume were calculated (Table 1). Also, the specific surface was calculated by the application of the BET model. ${ }^{[50]}$ The $\mathrm{N}_{2}$ adsorption-desorption isotherms shown in Figure $3 a$ (calcinated s0-3 nanoparticles) shows that pore filling occurred over a large range of $P / P_{0}$ indicating a wide pore size distribution. On the other hand, the first adsorption step appears at relative high pressure values $\left(0.7<\mathrm{P} / \mathrm{P}_{0}<0.8\right)$ evidencing the existence of large pores. This step preserves a type-IV isotherm but with a clear $\mathrm{H} 1$ hysteresis loop. The total specific area was calculated to be $527 \mathrm{~m}^{2} \mathrm{~g}^{-1}$, whereas the pore size distribution curve (Figure 3, inset) shows a very wide pore size distribution starting in $4 \mathrm{~nm}$ and extending up to $18.5 \mathrm{~nm}$, with a mean value of $c a$. $11.8 \mathrm{~nm}$. Futhermore, two shoulders at ca. 4.7 and $9.4 \mathrm{~nm}$ can also be appreciated.

On the other hand, a pore volume of $0.82 \mathrm{~cm}^{3} \mathrm{~g}^{-1}$ and surface area of $302 \mathrm{~m}^{2} \mathrm{~g}^{-1}$ where determined for solid S2 which means a lower $\mathrm{N}_{2}$ adsorption when compared with the starting calcined material. Moreover, two main features can be appreciated in the pore size distribution of solid S2. One is the shift of the maximum towards smaller size values, as it can be expected from the filling of the pores. The second one is that the distribution becomes narrower in the area of smaller pores. This observation may be related with lower difficulty for the FITC-Ins molecules to abandon the solid from the wider pores when washing solid $\mathbf{S 2}$ during the synthesis process. Hence, the smaller pores will remain fully filled while the big ones may still appear not completely blocked.
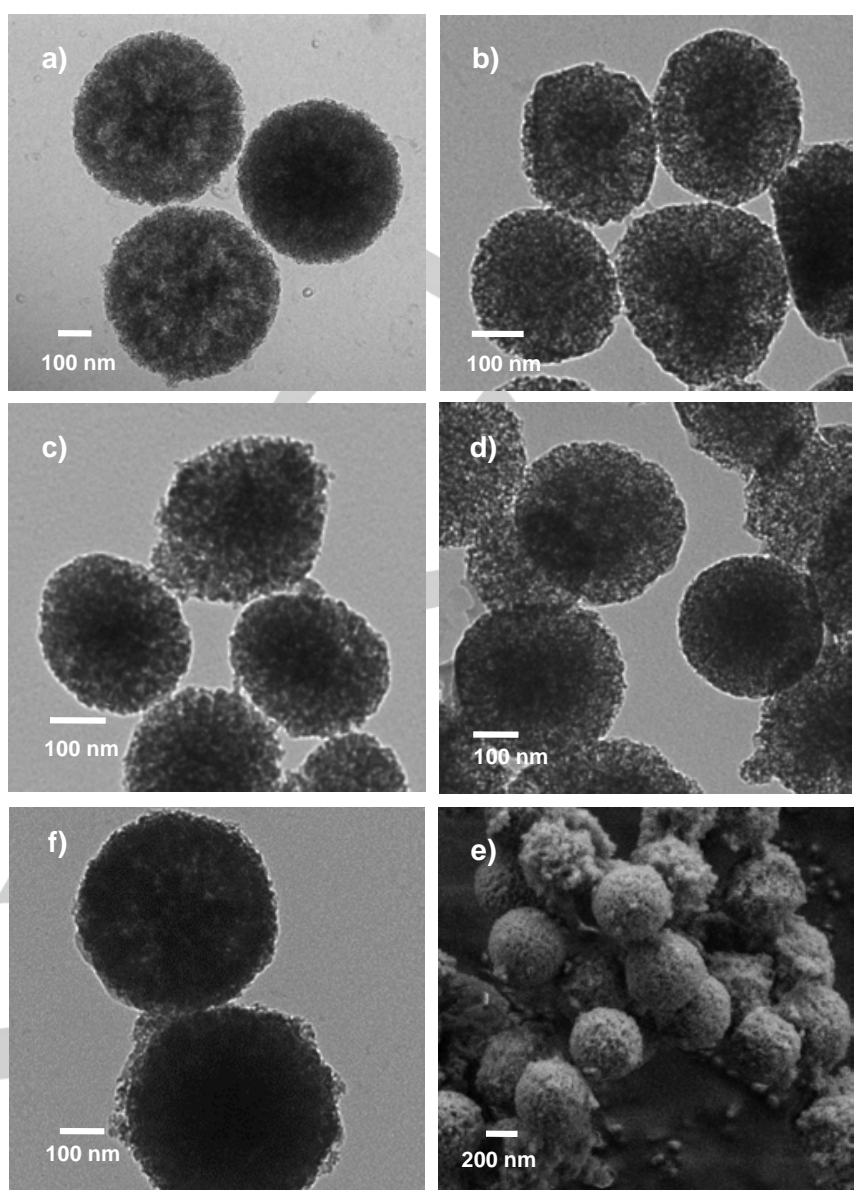

Figure 2. Representative TEM images of a) calcined nanoparticles, b) solid S1, c) solid S2, d) solid S3 and e) solid S4 showing the porosity of the silica-based nanomaterials. f) FSEM image of solid S3 showing the spherical morphology of the nanoparticles.

Table 1. BET specific surface values, pore volumes and pore sizes calculated from the $\mathrm{N}_{2}$ adsorption-desorption isotherms for selected materials.

\begin{tabular}{cccc}
\hline Sample & $\begin{array}{c}\mathrm{S}_{\mathrm{BET}} \\
\left(\mathbf{m}^{\mathbf{2}} \mathbf{g}^{-1}\right)\end{array}$ & $\begin{array}{c}\text { Pore volume } \\
\left(\mathbf{c m}^{\mathbf{3}} \mathbf{g}^{-\mathbf{1}}\right)\end{array}$ & $\begin{array}{c}\text { Pore diameter } \\
(\mathbf{n m})\end{array}$ \\
\hline S0-3 & 527 & 1.26 & 11.8 \\
S2 & 302 & 0.82 & 8.9 \\
\hline
\end{tabular}

The particle size studies were completed by measuring the diameter of calcined nanoparticles S0-3 and the hybrid materials S2 and S3 by dynamic light scattering (DLS) (Figure 4, and Table 2). All measurements were performed in triplicate on previously sonicated dispersions of the nanomaterials in simulated blood plasma at a concentration of $0.01 \mathrm{mg} \mathrm{mL}^{-1}$. The Z potential was also determined. Functionalization with benzimidazol moieties and loading with FITC-Ins reduced the zeta potential of the nanoparticles $\mathbf{S 2}(-26.5 \mathrm{mV})$ compared to that of calcined S0-3 $(-31.4 \mathrm{mV})$. For the final nanodevice S3, the surface charge was reduced even more (-17.7) compared to S2 as a consequence of capping with CD-GOx. In addition an increase of the hydrodynamic diameter after each 
functionalization step was observed which resulted in a hydrodynamic diameter of $426.1 \mathrm{~nm}$ for the final S3 nanodevice (see Table 2).
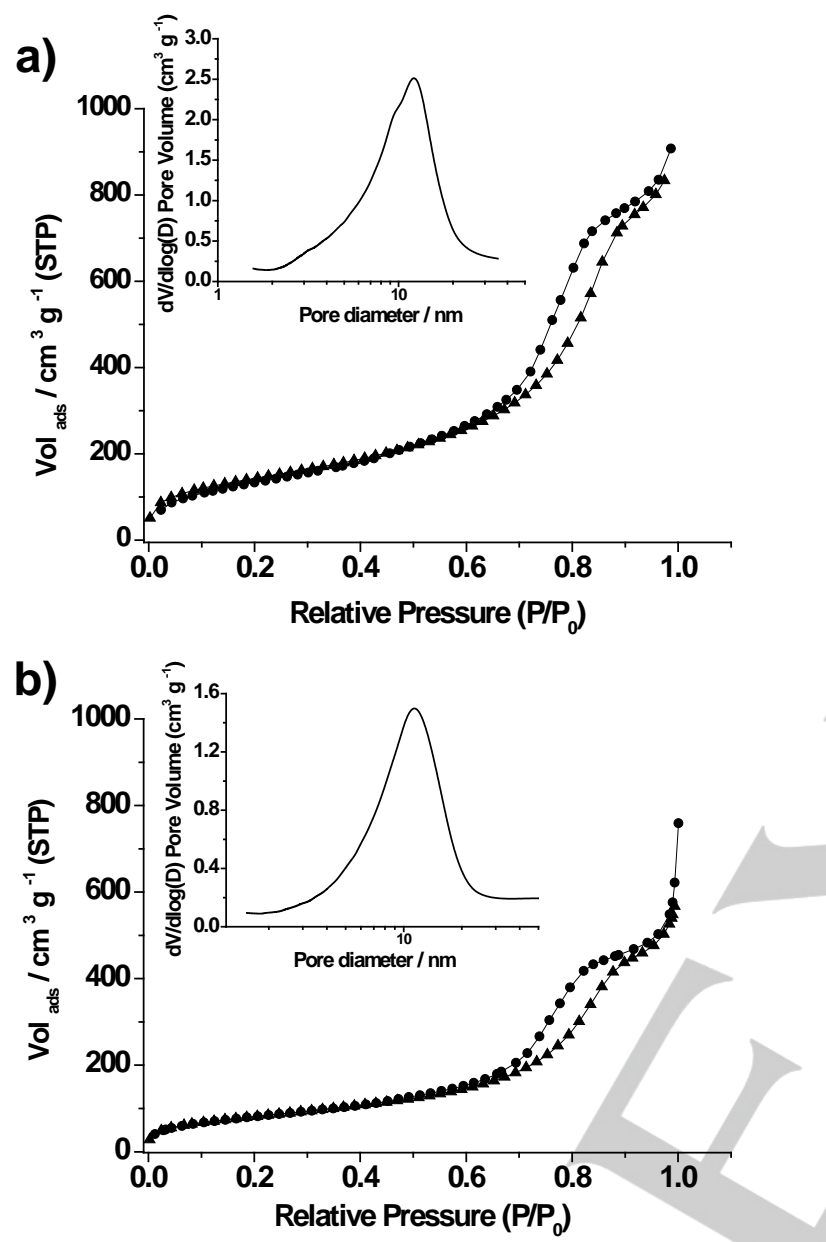

Figure 3. The nitrogen adsorption-desorption isotherms for a) calcined nanoparticles S0-3 and b) S2 material. Inset: pore size distribution of the calcined material.

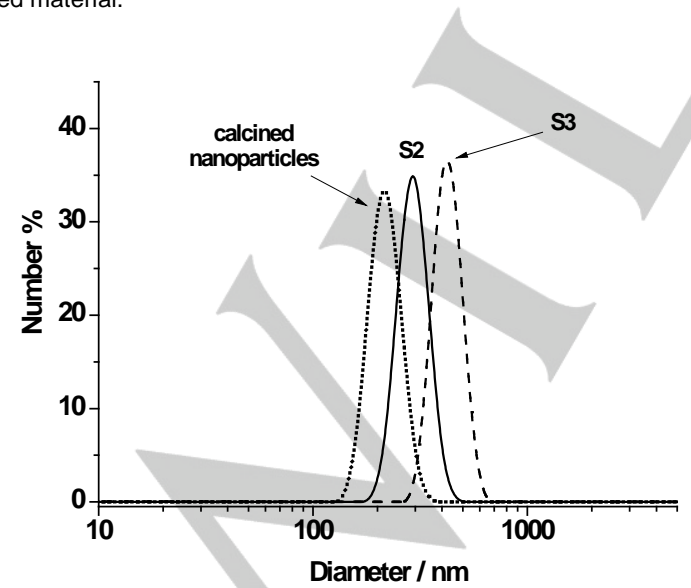

Figure 4. Particle size distribution by number of particles obtained by DLS studies for calcined nanoparticles S0-3, S2 and S3.
Table 2. Diameter measured by DLS and surface zeta potential of calcined nanoparticles S0-3, S2 and S3 materials.

\begin{tabular}{ccc}
\hline Sample & Particle diameter $(\mathbf{n m})$ & $\begin{array}{c}\text { Zeta potential } \\
(\mathbf{m V})\end{array}$ \\
\hline S0-3 & $237.9 \pm 30.3$ & -31.4 \\
S2 & $294.7 \pm 41.4$ & -26.5 \\
S3 & $426.1 \pm 54.2$ & -17.7 \\
\hline
\end{tabular}

Moreover, the content of 1-propyl-1-H-benzimidazole and FITC-Ins in S1, S2 and S3 were determined from thermogravimetric and elemental analysis and are detailed in Table 3. Besides, energy-dispersive X-ray spectroscopy (EDX) clearly showed the presence of $\mathrm{C}, \mathrm{N}, \mathrm{O}$ and $\mathrm{Si}$ atoms in these solids. In addition, with the aim to estimate the content of CDGOx retained in the final solid S3, the absorbance of CD-GOx in the solution after the capping process was measured. The amount of CD-GOx in the solid S3 was calculated to be $2.11 \cdot 10$ ${ }^{3} \mathrm{mg} \mathrm{g}^{-1} \mathrm{SiO}_{2}$, representing $2 \mathrm{U}$ of immobilized neoglycoenzyme per $\mathrm{mg} \mathrm{\textrm {SiO } _ { 2 }}$ according to enzyme activity assays.

Table 3. Content of FITC-Ins and 1-propyl-1- $\mathrm{H}$ - benzimidazole in the prepared solids $\mathbf{S} \mathbf{1}, \mathbf{S} 2$ and $\mathbf{S} 3$ in $\mathrm{mmol} \mathrm{g}^{-1} \mathrm{SiO}_{2}$.

\begin{tabular}{lcc}
\hline Sample & $\boldsymbol{\alpha}_{\text {FITC-Ins }}$ & $\boldsymbol{\alpha}_{\text {1-propyl-1-H-benzimidazole }}$ \\
\hline S1 & - & 1.24 \\
S2 & 0.20 & 1.24 \\
S3 & 0.17 & 1.24 \\
\hline
\end{tabular}

Glucose-triggered controlled release of insulin.

This section evaluates the self-regulated insulin delivery from S3 nanoparticles in the presence of glucose. As stated above, in humans, the concentration of this saccharide in blood is controlled by pancreas, which produces and delivers insulin in a finely regulated manner to maintain glucose between 3.5 and $7 \mathrm{mM}^{[24]}$ The assessment of $\mathbf{S 3}$ in a realistic competitive environment was accomplished by using simulated blood plasma. In a typical experiment, a suspension of $\mathbf{S} \mathbf{3}$ was added to simulated blood plasma ( $\mathrm{pH}$ 7.4) containing glucose at a concentration of $50 \mathrm{mM}$. At the same time a similar suspension of $\mathbf{S 3}$ in the absence of glucose was used as control. Both suspensions were stirred for $20 \mathrm{~h}$ under dark and aliquots were separated at programmed times and centrifuged in order to remove the nanoparticles and isolate the supernatant. FITC-Ins release was determined by monitoring the fluorescence intensity in the solution $\left(\lambda_{\mathrm{ex}}=495 \mathrm{~nm} ; \lambda_{\mathrm{em}}=514 \mathrm{~nm}\right)$. Figure 5 shows the delivery profile of FITC-Ins from solid S3 in the presence and in the absence of glucose as well as the the FITC-Ins efficiency release from S3 in \%. The results showed that solid S3 was tightly capped and only a $3 \%$ cargo was released after $20 \mathrm{~h}$ in the control experiment (see curve a in Figure 5). In contrast, the presence of $50 \mathrm{mM}$ of glucose induced a remarkable cargo 
delivery (ca. 26\% of loaded FITC-Ins) from solid S3 after $20 \mathrm{~h}$ (curve b). This observation confirms the proposed mechanism, in which the glucose oxidation to gluconic acid by the CD-GOX promotes cargo release. Also, it is worth mentioning that the spherical nanoparticle morphology and the porous structure persisted in solid $\mathbf{S 4}$ regardless of the FITC-Ins delivery (Figure 2f).

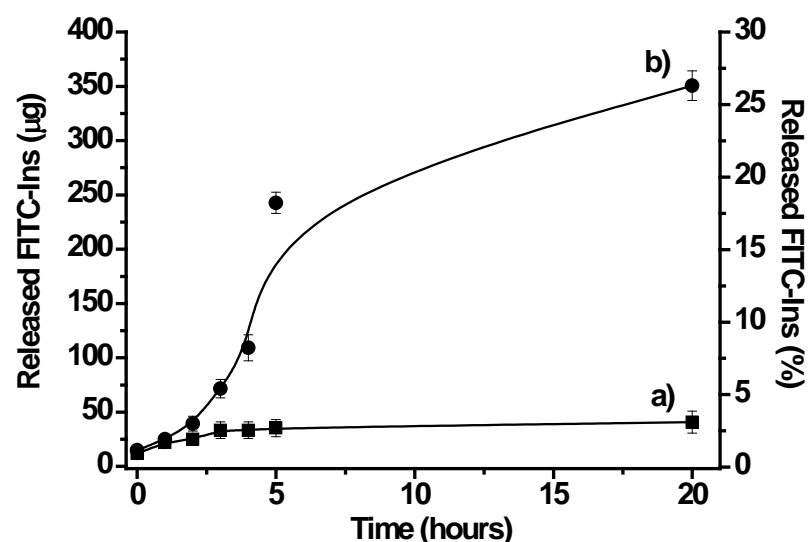

Figure 5. Amount of FITC-Ins released (left y axis) and FITC-Ins release efficiency (right $\mathrm{y}$ axis) from $1 \mathrm{mg}$ of solid $\mathbf{S 3}$ as a function of time in the absence (curve a) and in the presence of $50 \mathrm{mM}$ of glucose (curve b).

A more in depth, study of cargo release profile from $\mathbf{S 3}$ in the presence of glucose showed two different delivery trends. At shorter times, there was a quick release of insulin whereas at longer times the delivery was slower. In order to clarify these two different profiles the release kinetic in the presence of glucose at shorter times ( 0 to 4 hours) was fitted to the Higuchi model. Higuchi's model describes drug release as a diffusion process based in the Ficks's law and it is square root time dependent. ${ }^{[51]}$ This extended model takes into account the hypotheses that initial cargo concentration in the matrix is much higher than cargo solubility, that diffusion takes place in only one dimension and that cargo diffusivity is constant. This model has been broadly and satisfactorily applied to describe drug release kinetics from porous matrices. ${ }^{[52,53]}$ Figure 6 shows the fitting of the model to data taken in the first hours of the delivery $(0$ to $4 \mathrm{~h}$ ). The good fitting observed suggested that FITC-Ins delivery from S3 pores is essentially a diffusive process. At longer times a certain deviation from linearity was found (data not shown). This effect (two steps in the release profile) has also been described in other kinetic release studies of certain guests (usually drugs) from porous carrier materials.

As a step forward, the release of FITC-Ins from S3 as a function of the glucose concentration was studied. In these experiments FITC-Ins delivered after $4 \mathrm{~h}$ from $\mathbf{S} 3$ was studied in the glucose concentration range usually found in humans (i.e. up to $40 \mathrm{mM}$ ) (see Figure 7). The figure shows that in the presence of small amounts of glucose the delivered FITC-Ins is low. In contrast, when glucose concentration increased the capped system reacted delivering a high FITC-Ins dose mimicking healthy pancreas response.

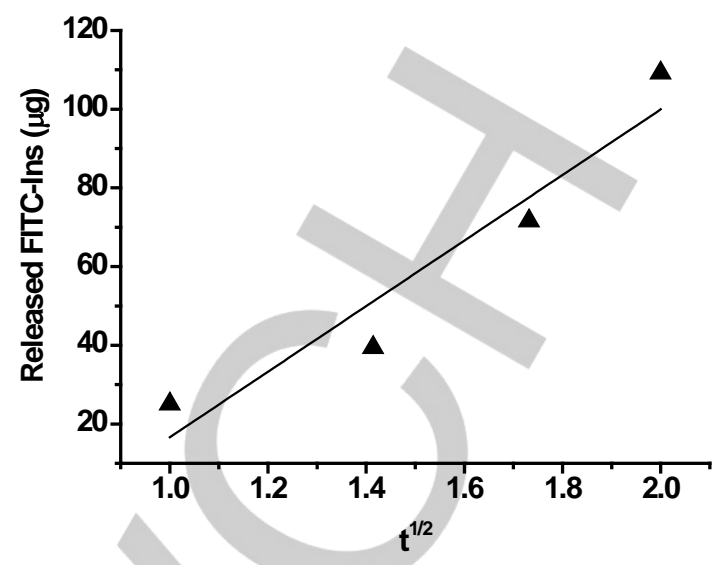

Figure 6. Amount of FITC-Ins delivered from solid S3 vs. the square root of time (t) in simulated human blood plasma.

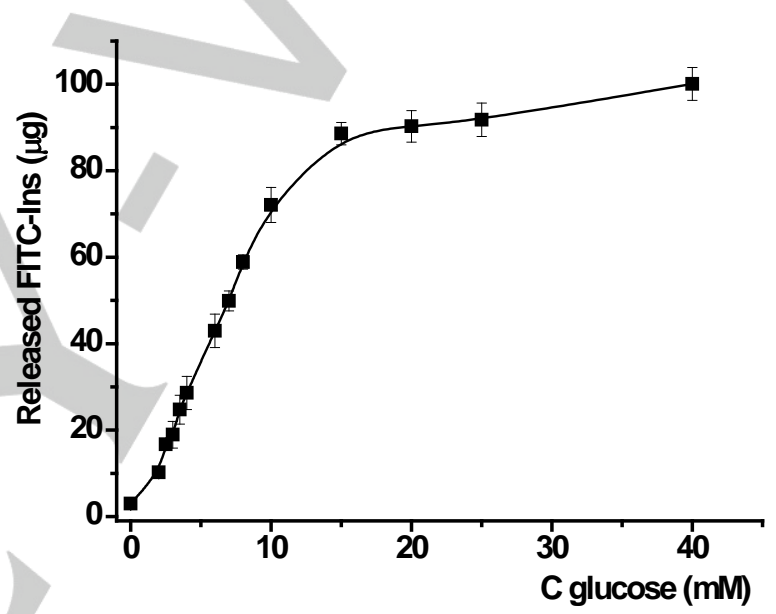

Figure 7. Amount of FITC-Ins released (left y axis) and FITC-Ins release efficiency (right y axis) from $1 \mathrm{mg}$ of solid S3 as a function of glucose concentration after $4 \mathrm{~h}$ upon addition of the nanomaterial.

The World Health Organization defines diabetes mellitus when fasting plasma glucose level is higher than $126 \mathrm{mg} / \mathrm{dL}(7.0$ $\mathrm{mM}$ ) after the intake of $75 \mathrm{~g}$ of glucose. ${ }^{[55]}$ This illness, if unmedicated, causes glucose peaks up to $40 \mathrm{mM}$, [56,57] especially after food intake. In a healthy person, to counteract this effect, blood insulin levels after eating rise more than 10 -fold from a baseline of around 20-30 pM to 250-300 pM. ${ }^{[24]}$ Taking into account that the average blood volume in an adult is $5 \mathrm{~L}$, and that S3 delivers ca. $5.91 \times 10^{-9} \mathrm{~mol}$ of Ins per $\mathrm{mg}$, (after 2.5 $\mathrm{h}$ for a concentration of glucose $50 \mathrm{mM}$ ) it can be estimated that the amount of S3 needed to reach insulin levels in the 250-300 $\mathrm{pM}$ range would be as low as ca. $210-233 \mu \mathrm{g}$ of S3 which is a quite low amount probably tolerable in an in vivo scenario.

An important issue when using delivery systems is that they should be ideally selective and deliver the cargo only in the presence of a unique target. In our case, when comparing meals of various combinations of sugars, proteins and fat, only glucose causes blood insulin levels to rise. ${ }^{[24]}$ Thus, it was in our aim to demonstrate that the CD-GOx capped material S3 is only opened in the presence of glucose and therefore control release experiments from $\mathbf{S} 3$ were carried out in the presence of other 
saccharides. In particular, the uncapping process in S3 was monitored after $4 \mathrm{~h}$ in simulated blood plasma containing glucose, mannose, fructose, galactose or saccharose at a concentration of $10 \mathrm{mM}$. As shown in Figure 8, S3 material shows a notable selectivity for glucose and FITC-Ins delivery from $\mathbf{S} 3$ in the presence of other saccharides was irrelevant. The selective triggering of the FITC-Ins delivery is ascribed to the ability of GOx to catalyze the oxidation of glucose to gluconic acid with high specificity.

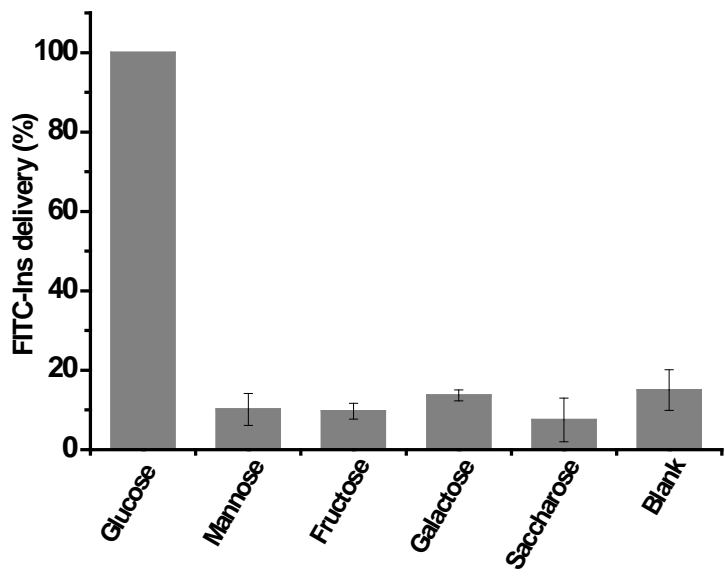

Figure 8. Release of FITC-Ins from solid S3 in the presence of different saccharides $(10 \mathrm{mM})$ after $4 \mathrm{~h}$.

\section{Conclusions}

In summary, we have developed a new glucose-sensitive system for insulin controlled release. Our system consists of MSNs with an expanded pore, loaded with FITC-Ins, functionalized in the external surface with 1-propyl-1-Hbenzimidazole groups and capped with CD-GOx. Conversion of glucose to gluconic acid by GOx induced the dethreading of the inclusion complexes and thus cargo release. The prepared material demonstrated to be glucose-sensitive by releasing FITC-Ins in simulated blood plasma. Moreover, the amount of delivered FITC-Ins was dependent of the concentration of glucose. Moreover, a relatively few amount of the material was able to release suitable amounts of insulin necessary to decrease the blood glucose concentration to the regular level. In addition, the system demonstrated to be highly selective and insulin delivery was only found with glucose whereas other saccharides were unable to uncap $\mathbf{5 3}$. This is one of the very few reported mesoporous supports able to release insulin triggered by the presence of glucose. Despite the fact that the road from these results to the use of nanoparticles to deliver insulin remains long and uncertain, we believe that such glucose-responsive nanoparticles able to detect levels of glucose in blood and deliver insulin accordingly may open new avenues to future designs of new drug-release system of potential application for diabetes treatment.

\section{Experimental Section}

General Techniques: Powder XRD, TG analysis, elemental analysis, TEM microscopy and $\mathrm{N}_{2}$ adsorption-desorption techniques were used to characterize the prepared materials. X-ray measurements were performed on a Bruker AXS D8 Advance diffractometer using Cu- $\mathrm{K}_{a}$ radiation. Thermo-gravimetric analysis were carried out on a TGA/SDTA 851e Mettler Toledo equipment, using an oxidant atmosphere (Air, 80 $\mathrm{mL} / \mathrm{min}$ ) with a heating program consisting on in a heating ramp of $10^{\circ}$ per minute from 273 to $373 \mathrm{~K}$ followed by an isothermal heating step at this temperature for $60 \mathrm{~min}$ in a nitrogen atmosphere $\left(80 \mathrm{~mL} \mathrm{~min}^{-1}\right)$. Then, the program was allowed to continue with a dynamic heating segment from 373 to $1273 \mathrm{~K}$ in an oxidant atmosphere (air, $80 \mathrm{~mL} \mathrm{~min}^{-1}$ ) and with an isothermal heating step at this temperature for $30 \mathrm{~min}$. TEM images were taken with a JEOL TEM-1010 Electron microscope working at 100 $\mathrm{kV}$. FSEM images and EDX analysis were performed with a Zeiss Ultra55 FESEM. $\mathrm{N}_{2}$ adsorption-desorption isotherms were recorded on a Micromeritics ASAP2010 automated sorption analyser. The samples were degassed at $120{ }^{\circ} \mathrm{C}$ in vacuum overnight. The specific surfaces areas were calculated from the adsorption data in the low pressures range using the BET model. Pore size was determined following the $\mathrm{BJH}$ method. Fluorescence spectroscopy was carried out on a Jasco FP-8300 Spectrometer.

Chemicals: The chemicals $n$-cetyltrimethylammonium bromide (CTAB), sodium hydroxide $(\mathrm{NaOH})$, fluorescein isothiocyanate (FITC), 3iodopropyltrimethoxysilane, tetramethylorthosilicate (TMOS), 1,3,5trimethylbenzene (TMB), benzimidazole, triethylamine, insulin human, dimethyl sulfoxide (DMSO), glucose, mannose, fructose, galactose, maltose and saccharose were provided by Aldrich. Analytical-grade solvents were provided by Scharlab. $\beta$-Cyclodextrin-modified glucose oxidase (CD-GOx) was synthesized as previously reported. ${ }^{[26]}$

Synthesis of MSNs with large pores S0-3: The synthetic procedure of MSNs with large pores was carried out according with previous reports with slight modifications. ${ }^{[21]} 3.94 \mathrm{~g}$ of $\mathrm{CTAB}$ and $2.28 \mathrm{~mL} 1 \mathrm{M}$ of $\mathrm{NaOH}$ were dissolved in $800 \mathrm{~g}$ ethanol/water $(0.4 / 0.6=\mathrm{w} / \mathrm{w})$. Then $1.3 \mathrm{~mL}$ of TMOS were added to the solution with vigorous stirring. After $8 \mathrm{~h}$, the mixture was kept unstirred overnight. The resulting white precipitate was isolated by centrifugation and washed 3 times with ethanol and 3 times with distilled water to obtain solid S0-1. The as-synthesised silica nanoparticles S0-1 were dispersed in $30 \mathrm{~mL}$ of ethanol and the mixture was sonicated for $30 \mathrm{~min}$. Subsequently, $20 \mathrm{~mL}$ of a 1:1 mixture (v/v) of water and TMB was added to the suspension. The mixture was kept in an autoclave at $140{ }^{\circ} \mathrm{C}$ for 4 days. The resulting solid S0-2 was isolated by centrifugation and washed 3 times with ethanol. Finally, the material S0-2 was calcined at $550{ }^{\circ} \mathrm{C}$ using oxidant atmosphere for $5 \mathrm{~h}$ in order to remove the organic content and obtain the support S0-3.

Synthesis of fluorescein isothiocyanate-labeled insulin (FITC-Ins): Insulin human was labelled with FITC following previous reports. ${ }^{[10]} 2.5$ $\mathrm{mL}$ of FITC in DMSO (1 mg mL-1$)$ was added in $5 \mu \mathrm{L}$ aliquots with gentle stirring to a sodium carbonate buffer $(0.1 \mathrm{M}, \mathrm{pH}$ 9) solution containing insulin ( $\left.4 \mathrm{mg} \mathrm{mL}^{-1}\right)$. The reaction was stirred for $2 \mathrm{~h}$ in dark and at room temperature. Then, $2.5 \mathrm{~mL}$ of $\mathrm{NH}_{4} \mathrm{Cl} 1 \mathrm{M}$ was added to the reaction in order to quench the excess of FITC. After stirring for an extra hour, the solution was dialyzed in phosphate-buffered saline and freeze dried yielding FITC labelled insulin. By measuring the absorbance at $495 \mathrm{~nm}$ and $280 \mathrm{~nm}$ the ratio of FITC to insulin was estimated at 1.4

Synthesis of S1: The hybrid solid S1 was prepared by adding an excess of 3-iodopropyltrimethoxysilane $(196 \mu \mathrm{L}, 1 \mathrm{mmol})$ to a suspension of 500 $\mathrm{mg}$ of $\mathbf{S 0 - 3}$. Then, the suspension was stirred for $5.5 \mathrm{~h}$. The resulting solid was filtered, washed with acetonitrile and dried at $70^{\circ} \mathrm{C}$ overnight. Following, $500 \mathrm{mg}$ of the resulting material were suspended in a $40 \mathrm{~mL}$ of a saturated solution of benzimidazole in toluene at $120^{\circ} \mathrm{C}$ and containing 
triethylamine (benzimidazol and triethylamine in a 1:3 proportion). The suspension was refluxed and stirred during $72 \mathrm{~h}$. The resulting white solid (S1) was filtered off, washed with $10 \mathrm{~mL}$ of acetonitrile and dried at $70{ }^{\circ} \mathrm{C}$ overnight.

Synthesis of S2: The loading of the pores was carried out by soaking $400 \mathrm{mg}$ of solid $\mathrm{S} 1 \mathrm{in} 40 \mathrm{~mL}$ of $\mathrm{HCl} 0.01 \mathrm{M}$ containing $200 \mathrm{mg}$ of the prepared FITC-Ins. The suspension was stirred for $24 \mathrm{~h}$ in dark. The resulting solid was filtered off to yield S2, washed with $2 \mathrm{~mL}$ of $\mathrm{HCl} 0.01$ $\mathrm{M}$, dried under vacuum and stored in the fridge.

Synthesis of S3: With the aim to obtain solid S3, $10 \mathrm{mg}$ of S2 were suspended in a CD-GOx solution ( $\left.1 \mathrm{~mL}, 1.56 \mathrm{mg} \mathrm{mL}^{-1}\right)$ and the suspension was stirred at room temperature for $24 \mathrm{~h}$. The final yellow solid (S3) was centrifuged (9500 rpm, $4 \mathrm{~min}, 20^{\circ} \mathrm{C}$ ) and washed with deionized water at $\mathrm{pH} 7.5$ five times in order to remove the residual dye and the free CD-GOx.

Release studies: In a typical experiment, $10 \mathrm{mg}$ of freshly prepared S3 solid were suspended in $1.5 \mathrm{~mL}$ of deionized water at $\mathrm{pH} 7.5$. Subsequently, the suspension was separated in different batches of 150 $\mu \mathrm{L}$ and each one was diluted to $3 \mathrm{~mL}$ with a mixture of simulated human blood plasma and deionized water at $\mathrm{pH}$ 7.5. At a certain time, an aliquot of $0.3 \mathrm{~mL}$ of the suspension was separated and centrifuged $(8000 \mathrm{rpm}, 3$ min). The delivered dye was registered by measuring the FITC-Ins emission in the supernatant $\left(\lambda_{\mathrm{ex}}=495 \mathrm{~nm}\right)$.

\section{Acknowledgements}

Authors thank the Spanish Government (projects CTQ201124355, MAT2015-64139-C4-1-R and AGL2015-70235-C2-2-R (MINECO/FEDER)) and the Generalitat Valenciana (project PROMETEOII/2014/047) for support. M.O. thanks the Universitat Politècnica de València for her FPI grant. P.D. thanks the Ministerio de Economía y Competitividad for her FPI grant (BES-2012-054066). C.C. thanks the Generalitat Valenciana for her postdoctoral contract VALi+D.

Keywords: Mesoporous silica $\bullet$ Glucose $・$ Insulin $•$ Controlled release $\bullet$ Molecular gates

[1] L. Nicole, C. Laberty-Robert, L. Rozes, C. Sanchez, Nanoscale 2014, 6, 6267-92.

[2] A. A. Beltrán-Osuna, J. E. Perilla, J. Sol-Gel Sci. Technol. 2015, 480496.

[3] B. G. Trewyn, I. I. Slowing, S. Giri, H. Chen, V. S. Lin, Acc. Chem. Res. 2007, 846-853.

[4] M. Vallet-Regí, F. Balas, Open Biomed. Eng. J. 2008, 2, 1-9.

[5] F. Sancenón, L. Pascual, M. Oroval, E. Aznar, R. Martínez-Máñez, ChemistryOpen 2015, 4, 418-437

[6] (a) E. Aznar, M. Oroval, L. Pascual, J. R. Murguía, R. Martínez-Máñez, F. Sancenón, Chem. Rev. 2016, 116 , 561-718; (b) E. Aznar, R. Martínez-Máñez, F. Sancenón, Exp. Opin. Drug Deliv. Rev. 2009, 6, 643-655.

[7] S. Alberti, G.J.A.A. Soler-Illia, O. Azzaroni, Chem. Commun. 2015, 51, 6050-6075.

[8] C. Argyo, V. Weiss, C. Bräuchle, T. Bein, Chem. Mater. 2014, 26, 435451.

[9] A. P. Wight, M. E. Davis, Chem. Rev. 2002, 102, 3589-3614.

[10] G. Kickelbick, Angew. Chem. Int. Ed. 2004, 43, 3102-3104.
[11] N. K. Mal, M. Fujiwara, Y. Tanaka, Nature 2003, 421, 350-353.

[12] J. Liu, C. Detrembleur, M.-C. De Pauw-Gillet, S. Mornet, C. Jérôme, E. Duguet, Small 2015, 11, 2323-2332.

[13] Q. Fu, G.V.R. Rao, L.K. Ista, Y. Wu, B.P. Andrzejewski, L.A. Sklar, T.L. Ward, G.P. Lopez, Adv. Mater. 2003, 15, 1262-1266.

[14] A. Baeza, E. Guisasola, E. Ruiz-Hernandez, M. Vallet-Regí, Chem. Mater. 2012, 24, 517-524.

[15] R. Hernandez, H.R. Tseng, J.W. Wong, J. F. Stoddart, J. I. Zink, J. Am. Chem. Soc. 2004, 126, 3370-3371.

[16] S. Niedermayer, V. Weiss, A. Hermann, A. Schmidt, S. Datz, K. Müller E. Wagner, T. Bein, C. Bräuchle, Nanoscale 2015, 7, 7953-7964.

[17] X. Zhang, F. Li, S. Guo, X. Chen, X. Wang, J. Li, Y. Gan, Biomaterials 2014, 35, 3650-3665.

[18] K. Patel, S. Angelos, W.R. Dichtel, A. Coskun, Y.W. Yang, J.I. Zink, J.F. Stoddart, J. Am. Chem. Soc. 2008, 130, 2382-2383.

[19] R. Bhat, A. Ribes, N. Mas, E. Aznar, F. Sancenón, M.D. Marcos, J.R. Murguía, A. Venkataraman, R. Martínez-Máñez, Langmuir 2016, 32, 1195-1200.

[20] C. Yu, L. Qian, M. Uttamchandani, L. Li, S.Q. Yao, S. Q. Angew. Chem. Int. Ed. 2015, 54, 10574-10578.

[21] M. Kavruk, O. Celikbickak, V.C. Ozalp, B.A. Borsa, F. Hernandez, G Bayramoglu, B. Salih, M.Y. Arica, Chem. Commun. 2015, 51, 8492 8495

[22] M.M Aye, S.L. Atkin, Drug Healthc. Patient Saf. 2014, 6, 55-67.

[23] L.-Y. Chu, Expert Opin. Ther. Patents. 2005, 15, 1147-1155.

[24] J. Suckale, M. Solimena, Frontiers Biosci. 2008, 13, 7156-7171.

[25] American Diabetes Association. Diabetes Care, 2014, 37, S14-S80.

[26] J. C. Pickup, F. Hussain, N. D. Evans, N. Sachedina, Biosens. Bioelectron. 2005, 20, 1897-1902.

[27] T. G. Farmer, T. F. Edgar, N. A. Peppas, J. Pharm. Pharmacol. 2008 $60,1-13$.

[28] G. P. Carino, E. Mathiowitz, Adv. Drug Deliv. Rev. 1999, 35, 249-257.

[29] K.A. Rubeaan, M. Rafiullah, S. Jayavanth, Expert Opin. Drug Deliv. 2016, 13, 223-237.

[30] A. Verma, N. Kumar, R. Malviya, P.K. Sharma, J Pharm. 2014, 2014 $1-9$.

[31] R. Mo, T. Jiang, J. Di, W. Taiac, Z. Gu, Chem. Soc. Rev. 2014, 43 3595-3629.

[32] K. Sato, Y. Imoto, J. Sugama, S. Seki, Langmuir 2005, 21, 797-799.

[33] S. Tanna, T. S. Sahota, K. Sawicka, M. J. Taylor, Biomaterials 2006, 27, 4498-4507.

[34] W. Qi, X. Yan, J. Fei, A. Wang, Y. Cui, J. Li, Biomaterials 2009, 30, 2799-2806.

[35] K. Ishihara, M. Kobayashi, N. Ishimaru, I. Shinohara, Polym. J. 1984 16, 625-631.

[36] Z. Wu, X. Zhang, H. Guo, C. Li, D. Yu, J. Mater. Chem. 2012, 22 22788.

[37] P. Liu, Q. Luo, Y. Guan, Y. Zhang, Polymer 2010, 51, 2668-2675.

[38] X. Zhang, Y. Guan, Y. Zhang, J. Mater. Chem. 2012, 22, 16299.

[39] N. Akhtar, S.A. El-Safty, M.E. Abdelsalam, H. Kawarada, Adv. Healthcare Mater. 2015, 4, 2110-2119.

[40] Y. Zhao, B. G. Trewyn, I. I. Slowing, V. S.-Y. Lin, J. Am. Chem. Soc 2009, 131, 8398-8400.

[41] W. Zhao, H. Zhang, Q. He, Y. Li, J. Gu, L. Li, H. Li, J. Shi, Chem Commun. 2011, 47, 9459-9461.

[42] R. N. Jain, X. Huang, S. Das, R. Silva, V. Ivanova, T. Minko, T. Asefa, Z. Anorg. Allg.Chem. 2014, 640, 616-623.

[43] É. Pérez-Esteve, A. Fuentes, C. Coll, C. Acosta, A. Bernardos, P. Amorós, M. D. Marcos, F. Sancenón, R. Martínez-Máñez, J. M. Barat, Micropor. Mesopor. Mater. 2015, 202, 124-132.

[44] C. Giménez, C. de la Torre, M. Gorbe, E. Aznar, F. Sancenón, J. R. Murguía, R. Martínez-Máñez, M. D. Marcos, P. Amorós, Langmuir 2015, 31, 3753-62.

[45] C. de la Torre, I. Casanova, G. Acosta, C. Coll, M.J. Moreno, F. Albericio, E. Aznar, R. Mangues, M. Royo, F. Sancenón, R. Martínez- 
Máñez, Adv. Funct. Mater. 2015, 25, 687-695

[46] E. Aznar, R. Villalonga, C. Giménez, F. Sancenón, M. D. Marcos, R. Martínez-Máñez, P. Díez, J. M. Pingarrón, P. Amorós, Chem. Commun. 2013, 49, 6391-6393.

[47] M. Mizutani, Y. Yamada, T. Nakamura, K. Yano, Chem. Mater. 2008, 20, 4777-4782.

[48] M. H. Kim, H. K. Na, Y. K. Kim, S. R. Ryoo, H. S. Cho, K. E. Lee, H. Jeon, R. Ryoo, D. H. Min, ACS Nano 2011, 5, 3568-3576.

[49] E. P. Barrett, L. G. Joyner, P. P. Halenda, J. Am. Chem. Soc. 1951, 73 373-380.

[50] S. Brunauer, P. H. Emmett, E. Teller, J. Am. Chem. Soc. 1938, 60 309-319.

[51] T. Higuchi, J. Pharm. Sci. 1963, 52, 1145-1149.

[52] É. Pérez-Esteve, M. Ruiz-Rico, C. De La Torre, L. A. Villaescusa, F. Sancenón, M. D. Marcos, P. Amorós, R. Martínez-Máñez, J. M. Barat, Food Chem. 2016, 196, 66-75.

[53] A. Bernardos, E. Aznar, C. Coll, R. Martínez-Mañez, J. M. Barat, M. D. Marcos, F. Sancenón, A. Benito, J. Soto, J. Control. Release 2008, 131, 181-189.

[54] K. Radhakrishnan, S. Gupta, D. P. Gnanadhas, P. C. Ramamurthy, D. Chakravortty, A. M. Raichur, Part. Part. Syst. Charact. 2014, 31, 449.

[55] World Health Organization: World Health Organization, International Diabetes Federation, editors. Definition and diagnosis of diabetes mellitus and intermediate hyperglycemia. Report of a WHO/IDF Consultation. Geneva (Switzerland): WHO Press; 2006.

[56] C.C. Thomas, L.H. Philipson, Med. Clin. N. Am. 2015, 99, 1-16.

[57] M. J. Mattu, and Gary W. Small, M. A. Arnold, Anal. Chem. 1997, 69, 4695. 


\section{Entry for the Table of Contents}

Layout 1:

\section{FULL PAPER}

A novel nanoplatform combining diagnosing and drug delivery capabilities is presented. The capped system is based on a nanometric silica support loaded with FITC-Ins and capped with CD-GOx. This nanosystem is able to release the entrapped FITC-Ins in a controlled manner due to the specific enzymatic reaction between glucose and GOx.

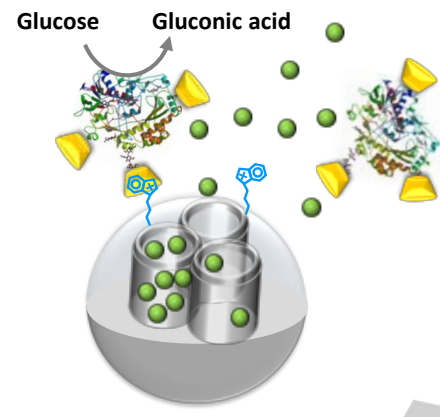

Mar Oroval, Paula Díez, Elena Aznar Carmen Coll, María Dolores Marcos, Félix Sancenón, Reynaldo Villalonga and Ramón Martínez-Máñez*

Page No. - Page No.

Self-regulated glucose-sensitive enzyme-capped mesoporous silica nanoparticles for insulin delivery

$$
O \Rightarrow \text { FITC-Ins }
$$

